\title{
Quasi-lines and their degenerations
}

\author{
LAURENT BONAVERO AND ANDREAS HÖRING
}

\begin{abstract}
In this paper we study the structure of manifolds that contain a quasiline and give some evidence towards the fact that the irreducible components of degenerations of the quasi-line should determine the Mori cone. We show that the minimality with respect to a quasi-line yields strong restrictions on fibre space structures of the manifold.
\end{abstract}

Mathematics Subject Classification (2000): 14E30 (primary); 14J10, 14J30, 14J40, 14J45 (secondary).

\section{Introduction}

Let $X$ be a complex quasiprojective manifold of dimension $n$. A quasi-line $l$ in $X$ is a smooth rational curve $f: \mathbb{P}^{1} \hookrightarrow X$ such that $f^{*} T_{X}$ is the same as for a line in $\mathbb{P}^{n}$, i.e. is isomorphic to

$$
\mathcal{O}_{\mathbb{P}^{1}}(2) \oplus \mathcal{O}_{\mathbb{P}^{1}}(1)^{\oplus n-1}
$$

Although the terminology suggests that quasi-lines are very special objects, we will see that they appear in a lot of situations.

\section{Examples 1.1.}

(1) If $X$ is a smooth Fano threefold of index 2 with $\operatorname{Pic}(X)=\mathbb{Z} H$, where $H$ is very ample, then a general conic $C$ (i.e. a curve satisfying $H \cdot C=2$ ) is a quasi-line (Oxbury [20], see also Bădescu, Beltrametti and Ionescu [3]).

(2) If $X$ is rationally connected, then there exists a sequence $X^{\prime} \rightarrow X$ of blow-ups along smooth codimension 2 centres such that $X^{\prime}$ contains a quasi-line (Ionescu and Naie [11]).

(3) If $X$ contains a quasi-line $l$, let $\pi: X^{\prime} \rightarrow X$ be a blow-up of $X$ along a smooth subvariety $Z$. A general deformation of $l$ does not meet $Z$, so it identifies to a quasi-line in $X^{\prime}$.

Received March 2, 2007; accepted September 5, 2007. 
Deformation theory shows that rational curves whose deformations passing through a fixed point dominate the manifold have $-K_{X}$-degree at least $\operatorname{dim} X+1$. Quasilines can be seen as the rational curves realising the boundary case $-K_{X} \cdot l=$ $\operatorname{dim} X+1$, so it is reasonable to ask if the existence of a quasi-line has any implications on the global structure of the manifold. Yet as the Example 1.1(2) shows this implication can't be much stronger than the rational connectedness of $X$, so we will have to make extra restrictions. The theory of Kählerian twistor spaces which provided the first motivation for the study of quasi-lines suggests that the most important class to study are Fano manifolds [10]. The cone theorem then shows that the Mori cone $\operatorname{NE}(X)$ of a Fano manifold is closed and polyhedral, the extremal rays being generated by classes of rational curves. If the Picard number is at least 2 , the class of a quasi-line does not generate an extremal ray, but we have the natural following question.

Question 1.2. For a Fano manifold $X$ containing a quasi-line $l$, do the numerical classes of irreducible components of degenerations of $l$ generate the Mori cone $\mathrm{NE}(X)$ ?

In this question, it is hopeless to expect that the numerical classes of irreducible components of a single degeneration of $l$ generate the Mori cone $\mathrm{NE}(X)$. Before asking such a question, one should better verify that degenerations exists. This is guaranteed by the characterisation of the projective space by Cho, Miyaoka and Shepherd-Barron, which we restate here in the language of quasi-lines (see Section 2 for the terminology).

Theorem $1.3([4,13])$. Let $X$ be a projective manifold that contains a quasi-line $l$. Suppose that for a general point $x$ of $X$, the deformations of $l$ passing through $x$ form an unsplit family of rational curves. Then $X \simeq \mathbb{P}^{n}$ and $l \subset \mathbb{P}^{n}$ is a line.

First evidence for an affirmative answer to the question comes from the following situation: suppose that there exists an effective divisor $D \subset X$ such that $D \cdot l=0$. Then there exists a degeneration that has an irreducible component in $D$, moreover there exists a birational Mori contraction whose locus is contained in $D$ (see Lemma 3.1). This observation leads us to recall the related notion of minimality with respect to a quasi-line, introduced by Ionescu et al. in $[3,12]$.

Definition 1.4. Let $X$ be a projective normal $\mathbb{Q}$-factorial (Fano) variety $X$ that contains a quasi-line $l$ in its nonsingular locus $X_{\mathrm{ns}}$ (we say that the couple $(X, l)$ is a (Fano) model). The variety $X$ is minimal with respect to $l$ if for every effective Cartier divisor $D \subset X$, we have $D \cdot l>0$. This also means that the numerical class of $l$ belongs to the interior of the cone generated by the classes of moving curves.

Two models $(X, l)$ and $\left(X^{\prime}, l^{\prime}\right)$ are equivalent if there are Zariski open subsets $U \subset X$ and $U^{\prime} \subset X^{\prime}$ containing respectively $l$ and $l^{\prime}$ and an isomorphism $\mu: U \rightarrow$ $U^{\prime}$ such that $\mu(l)=l^{\prime}$.

The notions of minimality and equivalent models were introduced in order to avoid situations like in Example 1.1(3), where $X^{\prime}$ is clearly not minimal. Our first main result is an inverse statement for smooth Fano models of dimension three. 
Theorem A. Let $(X, l)$ be a smooth Fano model of dimension three. Then there exists a birational morphism $\mu: X \rightarrow X^{\prime}$ onto a $\mathbb{Q}$-factorial projective threefold with at most terminal singularities such that $\mu$ is an equivalence of models $(X, l) \simeq\left(X^{\prime}, \mu(l)\right)$, and $X^{\prime}$ is minimal with respect to $\mu(l)$.

The core of this paper considers smooth models $(X, l)$ such that the manifold $X$ admits a fibration $\varphi: X \rightarrow Y$. The first task is to characterise situations where this induces a morphism of models $(X, l) \rightarrow(Y, \varphi(l))$, i.e. the image $\varphi(l) \subset Y_{\mathrm{ns}}$ is a quasi-line. Lemma 4.1 shows that the study of morphisms of models should be started by considering fibrations $\varphi: X \rightarrow \mathbb{P}^{1}$ such that $l$ is a section. We will investigate this in Subsection 4.1 and discover important differences between models with $e(X, l)=1$ (that is there exists exactly one deformation of $l$ through two general points of $X$, see Definition 2.2) and those with $e(X, l)>1$.

Theorem B. Let $(X, l)$ be a smooth Fano model of dimension at most four, and let $\varphi: X \rightarrow \mathbb{P}^{1}$ be a fibration such that $l$ is a section of $\varphi$. Then the general fibre $F$ contains a quasi-line. If furthermore $e(X, l)=1$, the variety $X$ is not minimal with respect to $l$.

If we accept the general idea that the extremal contractions of a Fano model $(X, l)$ are related to the degenerations of $l$, the minimality with respect to $l$ should also yield some restriction on the structure of the morphisms of $X$. An example for a birational morphism is the following characterisation of the projective space due to Bădescu, Beltrametti and Ionescu.

Theorem 1.5 (3, Theorem 4.4, Corollary 4.6). Let $(X, l)$ be a smooth model such that $X$ is minimal with respect to $l$. Suppose that there exists a big and nef divisor such that $H \cdot l=1$. Then $X \simeq \mathbb{P}^{n}$ and $l \subset \mathbb{P}^{n}$ is a line.

We will show a similar statement in a relative setting.

Theorem C. Let $\varphi:(X, l) \rightarrow(Y, \varphi(l))$ be a morphism of smooth models, and suppose that $\varphi$ is flat of relative dimension 1. Assume furthermore that:

(1) $X$ is minimal with respect to $l$ and $e(X, l)=1$,

(2) there exists a big and nef line bundle $H$ on $Y$ such that $\varphi^{*} H \cdot l=1$.

Then $Y \simeq \mathbb{P}^{n-1}$ and $X \simeq \mathbb{P}(E)$ where $E$ is a stable rank two vector bundle over $Y$. If furthermore $X$ is Fano, we have $\operatorname{dim} X=3$ and $X \simeq \mathbb{P}\left(T_{\mathbb{P}^{2}}\right)$.

This result follows from two intermediate results (Theorem 4.14 and Proposition 5.1) which are interesting in their own right and classification results of Fano bundles (Corollary 5.4 and Proposition 5.5).

Acknowledgements. We heartly thank Cinzia Casagrande and Rita Pardini for valuable discussions and explaining to us the very interesting Examples 4.15. We also thank Stéphane Druel for his proofreading and helping us to repair an error in the proof of Proposition 4.11. This work was done while the second named author 
held a post-doc position at IRMA Strasbourg. He wants to thank the members of the institute for their hospitality. This work has been partially supported by the $3 A G C$ project of the A.N.R.

\section{Notation and basic results}

We work over the complex field $\mathbb{C}$, topological notions always refer to the Zariski topology. A variety is an integral scheme of finite type over $\mathbb{C}$, a manifold is a smooth variety. A fibration is a surjective morphism $\varphi: X \rightarrow Y$ between normal varieties such that $\operatorname{dim} X>\operatorname{dim} Y>0$ and $\varphi_{*} \mathcal{O}_{X} \simeq \mathcal{O}_{Y}$, that is all the fibres are connected. Fibres are always scheme-theoretic fibres. For general definitions we refer to Hartshorne's book [8], we will also use the standard terminology of Mori theory and deformation theory as explained in $[5,15]$.

\subsection{Deformations and degenerations}

Let $X$ be a projective variety, and let $C \subset X$ be an integral projective curve. Identify $C$ to its fundamental cycle, ${ }^{1}$ and suppose that the Chow scheme $\mathcal{C}(X)$ is irreducible at the point $[C]$. Denote by $\mathcal{H}$ the normalisation of the unique irreducible component of $\mathcal{C}(X)$ containing $[C]$. Furthermore we have the incidence variety $\mathcal{U} \subset \mathcal{H} \times X$ endowed with two natural morphisms $q: \mathcal{U} \rightarrow \mathcal{H}$ and $p: \mathcal{U} \rightarrow X$, in particular $q$ is equidimensional.

Definition 2.1. A deformation $C^{\prime}$ of $C$ is an integral curve such that $\left[C^{\prime}\right] \in \mathcal{H}$. Let $h \in \mathcal{H}$ be a point such that the corresponding cycle $C_{h}$ is reducible or non-reduced, then $C_{h}$ will be called a degeneration of $C$. Denote by $\mathcal{H}^{*} \subset \mathcal{H}$ the open subset parametrizing deformations of $C$. The family $\mathcal{H}$ is said to be unsplit if $\mathcal{H}^{*}=\mathcal{H}$.

Let $Z \subset X$ be a subvariety. We say that the deformations of $C$ dominate (respectively cover) $Z$ if $p\left(q^{-1}\left(\mathcal{H}^{*}\right)\right) \cap Z$ is dense in $Z$ (respectively contains $Z$ ).

Let $(X, l)$ be a smooth model, and let $x \in l \subset X$ be a point. Since the normal bundle of $l$ is ample, the Chow scheme $\mathcal{C}(X)$ is smooth of dimension $2 \operatorname{dim} X-2$ at $[l]$. Therefore there exists a unique irreducible component $\mathcal{H}$ of $\mathcal{C}(X)$ containing $[l]$. The subscheme $q\left(p^{-1}(x)\right) \subset \mathcal{H}$ is smooth at $[l]$ and of dimension $n-1$. We denote by $\mathcal{H}_{x}$ the normalisation of the irreducible component of $q\left(p^{-1}(x)\right)$ that contains $[l]$. We denote by $\mathcal{U}_{x}$ the normalisation of the corresponding incidence variety in $\mathcal{H}_{x} \times X$. In order to ease the notation we denote the restriction of $p$ and $q$ to $\mathcal{U}_{x}$ by the same letter.

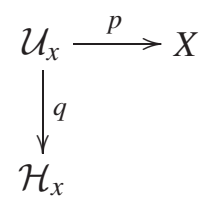

1 Throughout the whole paper, we will not distinguish between an effective cycle and its support. 
The general fibre of $q$ is a smooth $\mathbb{P}^{1}$ and its image in $X$ is still a quasi-line. Let $h \in \mathcal{H}_{x}$ be a point that parametrises a degeneration of $l$, then we'll denote the corresponding 1-cycle by $\sum_{i} \alpha_{i} l_{i}$ or $l \sim \sum_{i} \alpha_{i} l_{i}$, and call it a degeneration of $l$ with fixed point $x$.

One special feature of quasi-lines is that given a quasi-line and two general points, there exists finitely many deformations of the quasi-line passing through these two points. This fact is formalised in the next notion, introduced by Ionescu and Voica.

Definition 2.2. Let $(X, l)$ be a model, and let $x \in X$ be a general point. Let $p: \mathcal{U}_{x} \rightarrow X$ be the morphism from diagram 2.1, then we define

$$
e(X, l):=\operatorname{deg}(p)
$$

Since the evaluation morphism $p$ is surjective and generically finite, the number $e$ is well-defined. It is clear that $e$ is an invariant of Zariski equivalent models.

\subsection{A lemma}

The following technical lemma shows that if $e(X, l)=1$, the minimality with respect to $l$ yields a strong restriction on the degenerations of $l$.

Lemma 2.3. Let $(X, l)$ be a smooth model such that $e(X, l)=1$. Let $x \in X$ be a general point, and let $D \subset X$ be a prime effective divisor such that $x \notin D$. Suppose that through a general point of D passes an irreducible component $l_{i}$ of a degeneration $\sum_{i} \alpha_{i} l_{i}$ of $l$ with fixed point $x$ such that $l_{i} \subset D$. Then $D \cdot l=0$, in particular $X$ is not minimal with respect to $l$.

Proof. We use the notation of the basic Diagram 2.1. By definition $e(X, l)=1$ implies that the map $p: \mathcal{U}_{x} \rightarrow X$ is birational. We argue by contradiction and suppose that $D \cdot l>0$. Since $x \notin D$, this implies that for a general point $y \in$ $D$, there exists a quasi-line through $x$ and $y$. By hypothesis, there exists also a degeneration of $l$ through $y$. In particular $p^{-1}(y)$ is not a singleton, so $y$ is contained in the image of the exceptional locus of $p$. This is impossible since this image has codimension at least 2 .

\section{Minimality and birational contractions}

Question 1.2 asks if there are is a link between a quasi-line $l \subset X$ and the extremal contractions of $X$. If $X$ is minimal with respect to $l$, Section 4 will provide some evidence in special cases. If $X$ is not minimal, we can make a surprisingly simple observation formulated in the next lemma. Recall first that an elementary (Mori extremal) contraction $\varphi: X \rightarrow Y$ from a Fano manifold $X$ to a normal variety $Y$ is a morphism with connected fibres which contracts exactly the curves whose numerical class belong to a given extremal ray of the Mori cone $\mathrm{NE}(X)$ of $X$. 
Lemma 3.1. Let $(X, l)$ be a Fano model, and let $D$ be an effective prime divisor on $X$ such that $D \cdot l=0$. Then there exists a Mori contraction of birational type whose exceptional locus is contained in D. In particular, if all the elementary contractions of $X$ are of fibre type, then $X$ is minimal with respect to $l$.

Proof. Fix a general point $x \in X \backslash D$, then the deformations and degenerations of $l$ with fixed point $x$ cover $X$. Since $D \cdot l=0$, no deformation of $l$ meets $D$. For any point $y \in D$, we thus can write $l \sim \sum_{i=1}^{k} \alpha_{i} l_{i}$ with $\alpha_{i} \in \mathbb{N}^{*}$ such that one component passes through $y$. Since the cycle is connected and also passes through $x$, there exists a component $l_{i}$ that meets $D$ and is not contained in it, so $D \cdot l_{i}>0$. Therefore there exists another component, say $l_{1}$, that satisfies $D \cdot l_{1}<0$. Since $X$ is Fano we have a decomposition in $N_{1}(X) \otimes \mathbb{R}(c f$. [5, Chapter 1])

$$
l_{1}=\sum_{j} \beta_{j} \Gamma_{j},
$$

where the $\Gamma_{j}$ are generators of some extremal rays of the Mori cone $\mathrm{NE}(X)$ and $\beta_{j}>0$. Since $D \cdot l_{1}<0$ there exists at least one extremal rational curve $\Gamma_{j}$ such that $D \cdot \Gamma_{j}<0$. The corresponding extremal contraction has its exceptional locus contained in $D$, therefore it is birational.

Example 3.2. The only smooth model $(S, l)$ of dimension two that is minimal with respect to $l$ is the model $\left(\mathbb{P}^{2}\right.$, line $)$. Indeed consider the deformations of $l$ passing through a general point $x$. If $(S, l)$ is not $\left(\mathbb{P}^{2}\right.$, line $)$, there exists Theorem 1.3 at least one degeneration $\sum_{i=1}^{k} \alpha_{i} l_{i}$. Hence

$$
1=l \cdot l=l \cdot \sum_{i=1}^{k} \alpha_{i} l_{i}
$$

implies that $l \cdot l_{i}=0$ for some $i$. More generally if $(S, l)$ is a smooth model of dimension two, then $S$ is the blow-up of $\mathbb{P}^{2}$ at finitely many points, $l$ being the inverse image of a general line of $\mathbb{P}^{2}$ [12, Proposition 1.21].

In higher dimension the structure of the birational contractions $\mu: X \rightarrow X^{\prime}$ becomes more complicated. In particular the contraction might be small (which means that its exceptional locus in $X$ might be of codimension bigger than two). In this case $\mu(D) \subset X^{\prime}$ would be a Weil divisor such that $\mu(l)$ does not meet $\mu(D)$, thus contracting would not improve the situation and it is not clear if there exist an equivalent model which is minimal with respect to the quasi-line. The next theorem shows that everything works well in dimension three.

Theorem 3.3. Let $(X, l)$ be a smooth Fano model of dimension three. Then there exists a birational morphism $\mu: X \rightarrow X^{\prime}$ on a $\mathbb{Q}$-factorial projective threefold with at most terminal singularities such that $\mu$ is an equivalence of models $(X, l) \simeq\left(X^{\prime}, \mu(l)\right)$, and $X^{\prime}$ is minimal with respect to $\mu(l)$. 
Proof. Let $D_{1}, \ldots, D_{k}$ be the pairwise different effective prime divisors such that $D_{i} \cdot l=0$. Then by Lemma 3.1, there exists for every $i \in\{1, \ldots, k\}$ an elementary contraction of birational type $\mu_{i}: X \rightarrow X_{i}$ such that the exceptional locus is contained in $D_{i}$. Since birational contractions of smooth threefolds are divisorial the exceptional locus of $\mu_{i}$ is exactly $D_{i}$. Since $D_{i} \cdot l=0$, a general deformation of $l$ does not meet the exceptional locus, so the morphisms $\mu_{i}$ are equivalences of models.

By the classification of birational Mori extremal contractions in dimension 3 , the morphism $\mu_{i}$ contracts $D_{i}$ to a point, or $X$ is the blow-up of a manifold $X_{i}$ along a smooth curve. The model $X^{\prime}$ is obtained along the following algorithm.

Step 1. One of the morphisms $\mu_{i}$ is the blow-up of a Fano manifold $X_{i}$ along a smooth curve. We replace $(X, l)$ by the equivalent model $\left(X_{i}, \mu_{i}(l)\right)$ and restart the program.

Step 2. None of the morphisms $\mu_{i}$ is the blow-up of a Fano manifold $X_{i}$ along a smooth curve. It follows from [17, Proposition 4.5.] and [18] that for all $i$ the line bundle $\mathcal{O}_{D_{i}}\left(D_{i}\right)$ is negative on all the curves contained in $D_{i}$. More precisely, either $D_{i}$ is contracted to a point, or $D_{i}$ is isomorphic to $\mathbb{P}^{1} \times \mathbb{P}^{1}$ with normal bundle $\mathcal{O}_{D_{i}}\left(D_{i}\right) \simeq \mathcal{O}_{\mathbb{P}^{1} \times \mathbb{P}^{1}}(-1,-1)$ and $D_{i}$ is mapped to a smooth rational curve with normal bundle $\mathcal{O}_{\mathbb{P}^{1}}(-1) \oplus \mathcal{O}_{\mathbb{P}^{1}}(-1)$ (and in that case $X_{i}$ is smooth). A standard argument shows that $D_{i} \cap D_{j}=\emptyset$ for all $i \neq j$. We can then "do successively" the morphisms $\mu_{i}$, getting a birational morphism $\mu: X \rightarrow X^{\prime}$ such that the exceptional divisor is exactly $D_{1} \cup \ldots \cup D_{k}$. Moreover the variety $X^{\prime}$ is a $\mathbb{Q}$-Gorenstein projective threefold with at most terminal singularities since at each step, the morphism $\mu_{i}$ is a Mori contraction.

Finally, since a general quasi-line $l$ does not meet $D_{1} \cup \ldots \cup D_{k}$, the morphism $\mu$ is an equivalence of models $(X, l) \simeq\left(X^{\prime}, \mu(l)\right)$. Since the strict transform of any effective prime divisor on $X^{\prime}$ is an effective prime divisor on $X$, it is also clear that $X^{\prime}$ is minimal with respect to $\mu(l)$.

\section{Fibrations between models}

Let $(X, l)$ be a smooth model that admits a fibration $\varphi: X \rightarrow Y$. Since $l$ is a very free curve, it is clear that $l$ is not contracted by $\varphi$ and the image $\varphi(l)$ is also a very free curve in $Y$. The answer to the following questions is not so clear:

1. Do $Y$ or the general fibre $F$ contain quasi-lines? How are they related to $l$ ?

2. Does the minimality with respect to $l$ imply any restrictions on the fibre space structure?

In general one should not expect too much, but we will see that the restriction to morphisms of models $(X, l) \rightarrow(Y, \varphi(l))$ is a good framework for analysing these problems. 
Lemma 4.1. Let $\varphi: X \rightarrow Y$ a fibration from a projective manifold $X$ onto a projective manifold $Y$ of dimension at least 2.

(1) Let $l$ be a general deformation of a given quasi-line on $X$ and suppose that $\varphi(l)$ is smooth. Then the following are equivalent:

(a) the image $\varphi(l) \subset Y$ is a quasi-line,

(b) the curve $l \subset \varphi^{-1}(\varphi(l))$ is a quasi-line of the manifold $\varphi^{-1}(\varphi(l))$.

In this case, the morphism $l \rightarrow \varphi(l)$ is an isomorphism.

(2) Conversely, let $l$ be a rational curve in $X$ such that the image $\varphi(l) \subset Y$ is a quasi-line, the rational curve $l \subset \varphi^{-1}(\varphi(l))$ is a quasi-line of the manifold $\varphi^{-1}(\varphi(l))$ and the morphism $l \rightarrow \varphi(l)$ is an isomorphism. Then $l$ is a quasi-line of $X$.

Proof. Note first that since $\varphi(l)$ is general and smooth, the preimage $Z:=\varphi^{-1}(\varphi(l))$ is smooth of dimension $\operatorname{dim} X-\operatorname{dim} Y+1$. So we have an exact sequence

$$
0 \rightarrow N_{l / Z} \rightarrow N_{l / X} \rightarrow N_{Z / X} \otimes \mathcal{O}_{l} \rightarrow 0,
$$

and $N_{Z / X} \simeq \varphi^{*} N_{\varphi(l) / Y}$.

The statement (2) is then obvious since any extension

$$
0 \rightarrow \mathcal{O}_{\mathbb{P}^{1}}(1)^{\oplus a} \rightarrow E \rightarrow \mathcal{O}_{\mathbb{P}^{1}}(1)^{\oplus b} \rightarrow 0
$$

splits.

Let us show the statement (1): the isomorphism $N_{Z / X} \simeq \varphi^{*} N_{\varphi(l) / Y}$ implies

$$
\operatorname{deg} N_{Z / X} \otimes \mathcal{O}_{l}=d \cdot \operatorname{deg} N_{\varphi(l) / Y},
$$

where $d$ is the degree of the morphism $l \rightarrow \varphi(l)$. We have $\operatorname{deg} N_{l / X}=\operatorname{dim} X-1$ and $\varphi(l)$ is a very free curve, so $\operatorname{deg} N_{\varphi(l) / Y} \geq \operatorname{dim} Y-1 \geq 1$. Hence

$$
\begin{aligned}
\operatorname{deg} N_{l / Z} & =\operatorname{deg} N_{l / X}-\operatorname{deg} N_{Z / X} \otimes \mathcal{O}_{l} \\
& =\operatorname{dim} X-1-d \cdot \operatorname{deg} N_{\varphi(l) / Y} \\
& \leq \operatorname{dim} X-1-d \cdot(\operatorname{dim} Y-1) \\
& \leq \operatorname{dim} X-1-\operatorname{dim} Y+1=\operatorname{dim} Z-1,
\end{aligned}
$$

and equality holds if and only if $d=1$ and $\operatorname{deg} N_{\varphi(l) / Y}=\operatorname{dim} Y-1$.

(b) $\Rightarrow$ (a). If $l \subset Z$ is a quasi-line, then $\operatorname{deg} N_{l / Z}=\operatorname{dim} Z-1$, so equality holds in (*). Hence $\operatorname{deg} N_{\varphi(l) / Y}=\operatorname{dim} Y-1$ and $d=1$, so $\varphi(l)$ is a quasi-line and the morphism $l \rightarrow \varphi(l)$ is an isomorphism.

(a) $\Rightarrow$ (b). Suppose now that the image of a general quasi-line of $X$ is a quasi-line of $Y$. Fix a general point $x$ in $X$ such that for some quasi-line $l$ through $x$, the image $\varphi(l)$ is a quasi-line through $\varphi(x)$. Let $\mathcal{H}_{x}$ and $\mathcal{H}_{\varphi(x)}^{\prime}$ the corresponding varieties as defined in Section 2. Since $\varphi(l)$ is a quasi-line we have by [15, I, Theorem 6.8] a 
natural surjective morphism $\bar{\varphi}: \mathcal{H}_{x} \rightarrow \mathcal{H}_{\varphi(x)}^{\prime}$ defined by $[l] \mapsto\left[\varphi_{*}(l)\right]$. Choose $\left[l^{\prime}\right]$ such that $\bar{\varphi}^{-1}\left(\left[l^{\prime}\right]\right)$ has an irreducible component $S$ of $\operatorname{dimension} \operatorname{dim} X-\operatorname{dim} Y$ that parametrises at least one quasi-line. We may also assume that $Z:=\varphi^{-1}\left(l^{\prime}\right)$ is a smooth variety. By construction, $p\left(q^{-1}(S)\right) \subset Z$ (we use the notation of Diagram 2.1). Since $S$ parametrises a family of cycles through a fixed point $x$ that dominates $Z$ and a general member $l \in S$ is irreducible, it is a very free curve in $Z$. This shows that $\operatorname{deg} N_{l / Z} \geq \operatorname{dim} Z-1$. By the inequality (*) we even have equality, so $l$ is a quasi-line in $Z$.

Remark 4.2. The proposition shows that the study of fibrations $\varphi:(X, l) \rightarrow$ $(Y, \varphi(l))$, where $l$ and $\varphi(l)$ are quasi-lines, essentially reduces to the study of fibrations $\varphi:(X, l) \rightarrow \mathbb{P}^{1}$ where $l$ is both a quasi-line and a section of $\varphi$. Note however that in general, $\varphi^{-1}(\varphi(l))$ is not Fano even if $X$ and $Y$ are (see Example (2) immediately after Theorem 4.14). One question also immediately arises and will be studied in the next paragraph: if $l$ is a section of $\varphi:(X, l) \rightarrow \mathbb{P}^{1}$, what can we say about very free rational curves in the general fibre?

\subsection{Fibrations over a curve}

Recall that the pseudo-index of a Fano manifold $X$ is the positive integer defined as

$$
i_{X}:=\min \left\{-K_{X} \cdot C \mid C \text { is a rational curve of } X\right\} .
$$

This number has been very much studied in the last period, the general philosophy being that the Fano manifolds with high pseudo-index are the easiest to understand.

Lemma 4.3. Let $(X, l)$ be a smooth Fano model of dimension $n$, and let $\varphi: X \rightarrow$ $\mathbb{P}^{1}$ be a fibration such that $l$ is a section of $\varphi$. Then one of the following holds:

1. the pseudo-index of the general fibre is strictly smaller than $(n+1) / 2$,

2. the general fibre of $\varphi$ is isomorphic to $\mathbb{P}^{n-1}$.

If moreover $X$ is minimal with respect to $l$, the second case does not occur.

Proof. Fix a general point $x \in X$ such that $F:=\varphi^{-1}(\varphi(x))$ is a smooth fibre and such that there passes a quasi-line through $x$. Let $y \in F$ be a point different from $x$, then there exists no quasi-line through $x$ and $y$ : the quasi-line is a section, so it meets $F$ in exactly one point.

Therefore there exists a degeneration $\sum_{i=1}^{k} \alpha_{i} l_{i}$ connecting $x$ to $y$. Then we have $F \cdot l_{i} \geq 0$ for all $i$, and equality holds if and only if $l_{i}$ is contracted by $\varphi$. Since $l$ is a section, we have

$$
1=F \cdot l=F \cdot \sum_{i=1}^{k} \alpha_{i} l_{i}=\sum_{i=1}^{k} \alpha_{i} F \cdot l_{i} .
$$

It follows that there exists a unique component, say $l_{1}$ such that $F \cdot l_{1}=1$ and for $i>1$, we have $F \cdot l_{i}=0$. Since for $i>1$, the curves $l_{i}$ are contained in a $\varphi$-fibre, 
there exists a renumbering of $l_{2}, \ldots l_{k}$ and $2 \leq k^{\prime} \leq k$ such that for $2 \leq i \leq k^{\prime}$, the curve $l_{i}$ is contained in $F$ and the connected chain of curves $l_{2} \cup \ldots \cup l_{k^{\prime}}$ passes through $x$ and $y$.

We distinguish two cases:

a) there exists a $y \in F$, such that $k^{\prime}>2$. Denote by $i_{F}$ the pseudo-index of $F$, then

$$
n+1=-K_{X} \cdot l=-K_{X} \cdot\left(l_{1}+\sum_{i=2}^{k} \alpha_{i} l_{i}\right)>-K_{X} \cdot \sum_{i=2}^{k^{\prime}} \alpha_{i} l_{i} \geq 2 i_{F},
$$

so we are in the first case of our statement;

b) for all $y \in F \backslash\{x\}$, we have $k^{\prime}=2$. Then the curves $l_{2}$ form an unsplit family of curves that connects every point to $x$. It follows, by Theorem 1.3 that $F \simeq$ $\mathbb{P}^{n-1}$. Assume now that $X$ is minimal with respect to $l$. Since $l$ is a section, an intersection calculus shows that every fibre of $\varphi$ is integral. Hence $X$ is a projective bundle over $\mathbb{P}^{1}$ (by a simple refinement, due to Araujo, of Fujita's characterisation of projective bundles [6]). Finally we have $X \simeq \mathbb{P}\left(\mathcal{O}_{\mathbb{P}_{1}}^{\oplus n-1} \oplus\right.$ $\left.\mathcal{O}_{\mathbb{P}_{1}}(1)\right)$ by [12, Proposition 4.1], which is not minimal.

The next example shows that the lemma does not hold if the quasi-line is not a section.

Example 4.4. Let $X$ be a general member of $\left|\mathcal{O}_{\mathbb{P}^{1} \times \mathbb{P}^{n}}(1, d)\right|$, where $n, d$ are integers satisfying $n \geq 3$ and $1 \leq d \leq n$. Then $X$ is Fano and the general fibre of $\varphi: X \rightarrow \mathbb{P}^{1}$ is a smooth hypersurface of $\mathbb{P}^{n}$ of degree $d$ (hence with pseudo-index $n+1-d$ ). Moreover, $X$ contains a quasi-line $l$ (the inverse image of a general line in $\mathbb{P}^{n}$ ) but the map $\varphi_{\mid l}: l \rightarrow \mathbb{P}^{1}$ has degree $n$.

Theorem 4.5. Let $(X, l)$ be a smooth Fano model of dimension at most four, and let $\varphi: X \rightarrow \mathbb{P}^{1}$ be a fibration such that $l$ is a section of $\varphi$. Then the general fibre $F$ contains a quasi-line.

Proof. The statement is trivial for $\operatorname{dim} X=1,2$. If $\operatorname{dim} X=3$, the general fibre is a del Pezzo surface. All the del Pezzo surfaces except $\mathbb{P}^{1} \times \mathbb{P}^{1}$ contain quasi-lines, but the quadric is excluded by Lemma 4.3. If $\operatorname{dim} X=4$, the result is an immediate consequence of the Lemma 4.8 below which gives a much more precise information in this case: the quasi-line of $F$ is a component of a degeneration of $l$.

Before stating a technical lemma, recall that for any smooth quasi-projective variety $Y$, there exists a subset $Y^{\text {free }}$ which is the intersection of countably many dense open subsets of $Y$ such that any rational curve on $Y$ whose image meets $Y^{\text {free }}$ is free. 
Lemma 4.6. Let $F$ be Fano manifold of dimension at most three and let $x \in F$ be a fixed very general point. Assume that there is a connected chain of rational curves $l_{1}^{\prime} \cup \ldots \cup l_{k}^{\prime}$ passing through $x$ whose deformations cover a dense open subset of $F$ and such that $-K_{F} \cdot \sum_{i=1}^{k} l_{i}^{\prime} \leq \operatorname{dim} F+1$. Then a general deformation satisfies $k=1$ and $l_{1}^{\prime}$ is a very free rational curve in $F$ such that $-K_{F} \cdot l_{1}^{\prime}=\operatorname{dim} F+1$.

Proof. The statement is trivial is $F$ is a curve, so suppose $\operatorname{dim} F$ is 2 or 3 . We argue by contradiction and assume that for $y \in F$ a very general point, the connected chain of curves $\cup_{i=1}^{k} l_{i}^{\prime}$ passing through $x$ and $y$ is reducible, in particular $k \geq 2$. Note that since $x$ and $y$ are very general they are both in $F^{\text {free }}$. Furthermore we can suppose that no irreducible component $l_{i_{0}}^{\prime}$ contains both $x$ and $y$; otherwise the corresponding component $l_{i_{0}}^{\prime}$ would be very free [5, Proposition 4.20], so

$$
\operatorname{dim} F+1=-K_{F} \cdot \sum_{i} l_{i}^{\prime}>-K_{F} \cdot l_{i_{0}}^{\prime} \geq \operatorname{dim} F+1,
$$

a contradiction.

So up to renumbering we can suppose that $x \in l_{1}^{\prime}, y \in l_{2}^{\prime}$. Since $x \in F^{\text {free }}$, we have $-K_{F} \cdot l_{1}^{\prime} \geq 2$. Since $y \in F^{\text {free }}$, we also have $-K_{F} \cdot l_{2}^{\prime} \geq 2$. If $\operatorname{dim} F=2$, this contradicts $3 \geq-K_{F} \cdot \sum_{i} l_{i}^{\prime}$ and we are done. Suppose now that $\operatorname{dim} F=3$, then

$$
4 \geq-K_{F} \cdot \sum_{i=1}^{k} l_{i}^{\prime},
$$

so the preceeding inequalities imply $k=2$ and $-K_{F} \cdot l_{1}^{\prime}=-K_{F} \cdot l_{2}^{\prime}=2$. Since $x \in F^{\text {free }}$ there exist only finitely many rational curves $C$ such that $-K_{F} \cdot C=2$ and $x \in C$. Therefore there are at most finitely many curves $l_{1}^{\prime}$ passing through $x$, we denote their union by $L_{1}^{\prime}$. Since $l_{2}^{\prime}$ is free and $-K_{F} \cdot l_{2}^{\prime}=2$, we have $N_{l_{2}^{\prime} / F} \simeq \mathcal{O}_{\mathbb{P}^{1}}^{\oplus 2}$. It follows that $\operatorname{Hilb}(F)$ is smooth of dimension two at the point $\left[l_{2}^{\prime}\right]$ and we choose a smooth open neighbourhood $Z \subset \operatorname{Hilb}(F)$ parametrizing deformations of $l_{2}^{\prime}$ with the same normal bundle. Let $\Gamma$ be the universal family over $Z$, and let $p: \Gamma \rightarrow F$ and $q: \Gamma \rightarrow Z$ be the natural maps. Then $p$ is finite over its image and since $\operatorname{dim} L_{1}^{\prime}=1$ and $\operatorname{dim} Z=2$, this implies $q\left(p^{-1}\left(L_{1}^{\prime}\right) \subsetneq Z\right.$. Therefore a general chain of curves $l_{1}^{\prime} \cup l_{2}^{\prime}$ is not connected, a contradiction.

Remark 4.7. This lemma is the key ingredient for Lemma 4.8, and it is at this point that we use the hypothesis on the dimension. In fact the following example shows that Lemma 4.6 fails to hold already in dimension four: set $F:=\mathbb{P}\left(\mathcal{O}_{\mathbb{P}^{3}} \oplus \mathcal{O}_{\mathbb{P}^{3}}(3)\right)$ and denote by $\phi: F \rightarrow \mathbb{P}^{3}$ the natural projection. Then

$$
K_{F}=\phi^{*} \mathcal{O}_{\mathbb{P}^{3}}(-1) \otimes \mathcal{O}_{F}(-2)
$$

is antiample, so $F$ is Fano with Picard number two. The second Mori contraction $\psi: F \rightarrow F^{\prime}$ contracts the exceptional section of $\phi$ to a point and we denote by $E$ 
the exceptional divisor. Let $x$ and $y$ be arbitrary points in $F$, then the points can be joined by the connected rational cycle

$$
\phi^{-1}(\phi(x)) \cup \phi^{-1}(\phi(y)) \cup l_{x, y},
$$

where $l_{x, y}$ is the unique line in $E \simeq \mathbb{P}^{3}$ passing through $E \cap \phi^{-1}(\phi(x))$ and $E \cap$ $\phi^{-1}(\phi(y))$. Since $N_{E / F} \simeq \mathcal{O}_{\mathbb{P}^{3}}(-3)$, we immediately see that

$$
-K_{F} \cdot\left(\phi^{-1}(\phi(x))+\phi^{-1}(\phi(y))+l_{x, y}\right)=5=\operatorname{dim} F+1 .
$$

Yet by Proposition 5.3 below, the manifold $F$ does not contain a quasi-line.

Lemma 4.8. In the situation of the theorem, let $x \in X$ be a fixed very general point such that $x \in F^{\text {free }}$ for $F=\varphi^{-1}(\varphi(x))$ a smooth fibre. Then there exists a quasiprojective $(\operatorname{dim} X-2)$-dimensional family of degenerations of $l$ with fixed point $x$ that are of the form

$$
l_{1}+l_{2}
$$

where $l_{1}$ is a section of $\varphi$ such that $-K_{X} \cdot l_{1}=1$ and $l_{2} \subset F=\varphi^{-1}(\varphi(x))$ is a very free rational curve in $F$ such that $-K_{F} \cdot l_{2}=\operatorname{dim} F+1$. If $\operatorname{dim} F=3$, the

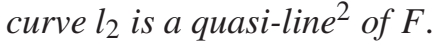

Proof. We assume that $\operatorname{dim} X=3$ or 4 , the other cases being trivial.

As seen in the proof of Lemma 4.3, for all $y \in F \backslash\{x\}$ the degenerations of $l$ through $x$ that connect $x$ to $y$ are of the form

$$
l_{1}+\sum_{i=2}^{k^{\prime}} \alpha_{i} l_{i}+\sum_{i=k^{\prime}+1}^{k} \alpha_{i} l_{i},
$$

where $l_{1}$ is a section of $\varphi$, the connected chain of curves $\sum_{i=2}^{k^{\prime}} \alpha_{i} l_{i}$ is contained in $F$ and passes through $x$ and $y$. By Lemma 4.6 the connected chain of curves $\cup_{i=2}^{k^{\prime}} l_{i}$ passing through $x$ and $y$ is irreducible, so $k^{\prime}=2$ and $l_{2}$ contains $x$ and $y$. By the same lemma for $y \in F$ very general, the curve $l_{2}$ is very free in $F$ and $-K_{F} \cdot l_{2}=\operatorname{dim} F+1$. Since $y$ varies in a family whose closure has dimension $\operatorname{dim} X-1$, we obtain a $(\operatorname{dim} X-2)$-dimensional family of degenerations having the stated structure.

Theorem 4.9. Let $(X, l)$ be a smooth Fano model of dimension at most four, and let $\varphi: X \rightarrow \mathbb{P}^{1}$ be a fibration such that $l$ is a section of $\varphi$. If $e(X, l)=1$, then the variety $X$ is not minimal with respect to $l$.

2 By [15, II, Theorem 3.14] a general deformation of a very free curve is an embedding if the ambient variety has dimension at least three, but only an immersion if the dimension is two. Recall that we defined quasi-lines to be smooth curves. 
Proof. The case $\operatorname{dim} X=2$ is treated in Example 3.2, suppose now that $\operatorname{dim} X \geq 3$. Fix a general point $x \in X$ and consider the closure in $\mathcal{C}(X)$ of the family given by Lemma 4.8. We obtain a $(\operatorname{dim} X-2)$-dimensional family $S$ of degenerations of $l$ with fixed point $x$ such that the general degeneration is of the form $l_{1}+l_{2}$, where $l_{1}$ is a section of $\varphi$ with $-K_{X} \cdot l_{1}=1$ and $l_{2} \subset F=\varphi^{-1}(\varphi(x))$ is a very free rational curve in $F$ with $-K_{F} \cdot l_{2}=\operatorname{dim} F+1$. Let $\Gamma$ be the normalization of the incidence variety, and let $p: \Gamma \rightarrow X$ and $q: \Gamma \rightarrow S$ be the natural maps. By construction the fibre $F:=\varphi^{-1}(\varphi(x))$ is contained in $p(\Gamma)$, but does not contain the curves $l_{1}$. Since $F$ is a divisor this shows that $p(\Gamma)$ has at least two irreducible components, that is the fibre $F$ and a component $D$ that contains the curves $l_{1}$. We claim that $D$ is a divisor that is dominated by the deformations of $l_{1}$. Assume this for moment, then $-K_{X} \cdot l_{1}=1$ implies that the deformations of $l_{1}$ form an unsplit family, so they cover $D$. This implies that $x \notin D$, otherwise there would exist a non-free curve passing through $x$. Conclude with Lemma 2.3.

Proof of the claim. We argue by contradiction and suppose that the locus $V \subset D$ dominated by the curves $l_{1}$ is not a divisor. Since $V \not \subset F$, the intersection $V \cap F \subset F$ is not a divisor. Since the chain $l_{1} \cup l_{2}$ is connected and $l_{1} \cap l_{2} \subset V \cap F$, all the curves $l_{2}$ pass through $V \cap F$ and $x$. This is impossible by [15, II, Proposition 3.7].

Remark 4.10. The proof above uses the hypothesis on the dimension of $X$ only to assure the existence of a family of degenerations of the form $l_{1}+l_{2}$.

The next proposition shows how one can recover a quasi-line by smoothing degenerations of the form $l_{1}+l_{2}$.

Proposition 4.11. Let $X$ be a Fano manifold of dimension $n$, and let $\varphi: X \rightarrow \mathbb{P}^{1}$ be an elementary contraction such that the general fibre $F$ contains a quasi-line $l_{2}$, that is

$$
\left.T_{F}\right|_{l_{2}} \simeq \mathcal{O}_{\mathbb{P}^{1}}(2) \oplus \mathcal{O}_{\mathbb{P}^{1}}(1)^{\oplus n-2}
$$

Suppose furthermore that the second elementary contraction $\psi: X \rightarrow Y$ has length 1 , and that a generator $l_{1}$ of the corresponding extremal ray is a section of $\varphi$. Then $X$ contains a quasi-line that is a section of $\varphi$.

\section{Proof.}

Step 1. Since the second contraction $\psi$ has length 1 , it is either a conic bundle (with singular fibres) or $X$ is a blow-up of the smooth variety $Y$ along a smooth subvariety of codimension two [23, Corollary 1.4]. In both cases, there exists an irreducible divisor $D \subset X$ that is dominated by the deformations of a generator $l_{1}$ of the second extremal ray and

$$
\left.T_{X}\right|_{l_{1}} \simeq \mathcal{O}_{\mathbb{P}^{1}}(2) \oplus \mathcal{O}_{\mathbb{P}^{1}}^{\oplus n-2} \oplus \mathcal{O}_{\mathbb{P}^{1}}(-1) .
$$


Fix now a general point $x \in X \backslash D$ such that $F=\varphi^{-1}(\varphi(x))$ is smooth and the quasi-lines $l_{2}$ passing through $x$ dominate $F$. Since $\varphi$ is an elementary contraction of fibre type, the divisor $D$ is strictly positive on the first extremal ray, in particular $(D \cap F) \cdot l_{2}=D \cdot l_{2}>0$. Hence for a general curve $l_{1}$ there exists a quasi-line $l_{2}$ such that $C:=l_{1} \cup l_{2}$ is connected. Since $l_{1}$ and $l_{2}$ are smooth curves, the intersection $l_{1} \cap l_{2}=l_{1} \cap F$ is a reduced point. The connected curve $C$ is a tree of rational curves and we will follow closely the argument in [5, Proposition 4.24] to show that $C$ is smoothable fixing the point $x$ (we refer to [5, Chapter 2] for the notation and details on schemes parametrizing morphisms). Consider the inclusion morphism $f: C \rightarrow X$ and let $b \in l_{2}$ be the unique point such that $f(b)=x$. Let $\pi: \mathcal{C} \rightarrow(T, 0)$ be a smoothing ${ }^{3}$ of the tree $C$ and let $\sigma: T \rightarrow \mathcal{C}$ be a section such that $\sigma(0)=b$. Denote by $g: \sigma(T) \rightarrow X \times T$ the morphism defined by $g(\sigma(t))=(b, t)$. The $T$-morphisms from $\mathcal{C}$ to $X \times T$ extending $g$ are parametrised by the $T$-scheme $\operatorname{Mor}_{T}(\mathcal{C}, X \times T ; g)$ whose fibre at 0 is $\operatorname{Mor}(C, X, b \mapsto x)$. By [15, II, Theorem 1.7] the irreducible components of $\operatorname{Mor}_{T}(\mathcal{C}, X \times T ; g)$ at $[f]$ have dimension at least

$$
\chi\left(C, f^{*} T_{X}(-b)\right)+\operatorname{dim} T .
$$

Step 2. We claim that $\operatorname{Mor}(C, X, b \mapsto x)$ has a component of the expected dimension, that is equal to $\chi\left(C, f^{*} T_{X}(-b)\right)$.

The exact sequence

$$
0 \rightarrow f^{*} T_{X}\left(-b-\left(l_{1} \cap l_{2}\right)\right) \otimes \mathcal{O}_{l_{2}} \rightarrow f^{*} T_{X}(-b) \rightarrow f^{*} T_{X}(-b) \otimes \mathcal{O}_{l_{1}} \rightarrow 0 .
$$

immediately implies $\chi\left(C, f^{*} T_{X}(-b)\right)=n+1$. Moreover the general fibres of the restriction morphism $\operatorname{Mor}(C, X, b \mapsto x) \rightarrow \operatorname{Mor}\left(l_{1}, X\right)$ consist of quasi-lines of $F$ passing through two fixed points, hence are 0-dimensional. Therefore there exists at least one component $Z \subset \operatorname{Mor}(C, X, b \mapsto x)$ that dominates $\operatorname{Mor}\left(l_{1}, X\right)$ and its dimension is equal to

$\operatorname{dim} \operatorname{Mor}\left(l_{1}, X\right)=h^{0}\left(l_{1}, f^{*} T_{X} \otimes \mathcal{O}_{l_{1}}\right)=h^{0}\left(l_{1}, \mathcal{O}_{\mathbb{P}^{1}}(2) \oplus \mathcal{O}_{\mathbb{P}^{1}}^{\oplus n-2} \oplus \mathcal{O}_{\mathbb{P}^{1}}(-1)\right)=n+1$.

Therefore the fibre at 0 of the morphism $\operatorname{Mor}_{T}(\mathcal{C}, X \times T ; g) \rightarrow T$ has a component of dimension $\chi\left(C, f^{*} T_{X}(-b)\right)$. Since the components of $\operatorname{Mor}_{T}(\mathcal{C}, X \times T ; g)$ have dimension at least $\chi\left(C, f^{*} T_{X}(-b)\right)+\operatorname{dim} T$, it follows that $\operatorname{Mor}_{T}(\mathcal{C}, X \times T ; g)$ has a component of the expected dimension $\chi\left(C, f^{*} T_{X}(-b)\right)+\operatorname{dim} T$ that dominates $T$, hence the morphism $\operatorname{Mor}_{T}(\mathcal{C}, X \times T ; g) \rightarrow T$ is dominant at $[f]$. In particular there exists in $\operatorname{Mor}_{T}(\mathcal{C}, X \times T ; g)$ an irreducible (open) curve passing through $[f]$ that dominates $T$. Denote by $T^{\prime}$ its normalization, then the morphism $T^{\prime} \rightarrow \operatorname{Mor}_{T}(\mathcal{C}, X \times T ; g)$ yields a $T^{\prime}$-morphism

$$
\mathcal{C} \times_{T} T^{\prime} \rightarrow X \times T^{\prime}
$$

which is the smoothing to a rational curve $l$ keeping fixed $f(b)=x$.

${ }^{3}$ Such a smoothing always exists and we can take $T$ to be the unit disc in $\mathbb{C}, c f$. [5, page 101]. 
Step 3. Clearly $-K_{X} \cdot l=n+1$ and $F \cdot l=F \cdot l_{1}+F \cdot l_{2}=1$. If we show that the deformations of $l$ keeping fixed $x$ dominate $X$, we conclude by [5, Proposition 4.20] that a general deformation of $l$ is very free, so it is a quasi-line. We argue by contradiction and suppose that this is not the case. Then the locus $V$ of deformations and degenerations of $l$ is a finite union of proper subvarieties, and the divisors $F$ and $D$ are irreducible components of this locus. Choose now a connected cycle $l_{1} \cup l_{2}$ that is not contained in any irreducible component of $V$. Since $l_{1} \cup l_{2}$ is smoothable by some deformation $l$, we can choose an irreducible curve $l^{\prime}$ arbitrarily close to $l_{1} \cup l_{2}$ that is contained in $V$. Yet this implies that $l^{\prime} \subset F \cup D$, so by irreducibility of $l^{\prime}$ we have $l^{\prime} \subset F$ or $l^{\prime} \subset D$. Therefore we have $\left(l_{1} \cup l_{2}\right) \subset F$ or $\left(l_{1} \cup l_{2}\right) \subset D$ which is a contradiction.

\subsection{New examples}

In this section only, $X$ is a Fano threefold with Picard number 2 which has a fibration $\varphi: X \rightarrow \mathbb{P}^{1}$ and a quasi-line $l$ being a section of $\varphi$. Since $X$ has Picard number 2 , there is another Mori extremal contraction $\psi: X \rightarrow Y$, whose fibres have dimension at most one, hence either $\psi$ is a conic bundle, or $\psi$ is the blow-up of the smooth variety $Y$ with centre a smooth curve. We will deal with the case of conic bundles in the Subsection 4.3. In the case where $\psi$ is the blow-up of a smooth variety $Y$ with centre a smooth curve, the situation is very nice: $\psi$ is determined by the degenerations of $l$.

Corollary 4.12. Let $(X, l)$ be a smooth Fano model of dimension three that admits an elementary contraction $\varphi: X \rightarrow \mathbb{P}^{1}$ such that $l$ is a section of $\varphi$. Assume that the second elementary Mori contraction is a birational contraction $\psi: X \rightarrow$ $Y$. Then both extremal rays of $\mathrm{NE}(X)$ are spanned by irreducible component of degenerations of $l$.

Proof. By Lemma 4.8 there exists a positive-dimensional quasi-projective family of degenerations of $l$ with fixed point a general point $x$ that are of the form

$$
l_{1}+l_{2}
$$

where $l_{1}$ is a section of $\varphi$ such that $-K_{X} \cdot l_{1}=1$ and $l_{2} \subset F=\varphi^{-1}(\varphi(x))$ satisfies $-K_{F} \cdot l_{2}=3$. In particular, the extremal ray defining $\varphi$ is spanned by $l_{2}$.

Let $C$ be a generator of the extremal ray contracted by $\psi$, then $F \cdot C=1$ by [16, Proposition 6]. Let $E$ be the exceptional divisor of $\psi$, then there exists rational numbers $a$ and $b$ such that $E=a\left(-K_{X}\right)+b F$. Hence

$$
\begin{aligned}
E \cdot C=-1 & =a+b \\
E \cdot l & =4 a+b \\
E \cdot l_{2} & =3 a .
\end{aligned}
$$

This implies $E \cdot l_{1}=E \cdot l-E \cdot l_{2}=a+b=-1=E \cdot C$. Since furthermore $-K_{X} \cdot l_{1}=1=-K_{X} \cdot C$ and $X$ has Picard number 2, this implies that $l_{1}$ and $C$ 
have the same numerical class. It follows that $l_{1}$ is contracted by $\psi$, so it generates the second extremal ray.

Examples 4.13. Under the assumptions of the corollary, and using again $[16,17]$, the threefold $Y$ is Fano of index $r \geq 2$ and easy computations show that $a=r-1$, therefore $b=-r$ and $E \cdot l=3 r-4$. Moreover, since $E \cdot l_{1}=1$, the morphism $\left.\psi\right|_{F}: F \rightarrow F^{\prime}=\psi(F)$ is an isomorphism for any general $\varphi$-fibre $F$ and $F^{\prime} \in\left|(r-1) \mathcal{O}_{Y}(1)\right|$. Moreover, we know (by $[16,17]$ again) that $Y$ is either $\mathbb{P}^{3}, \mathcal{Q}_{3}$ or one of the five Fano threefolds $V_{d}(1 \leq d \leq 5)$ of index 2 and Picard number 1 . Here are three explicit examples:

(1) Let $\psi: X \rightarrow \mathbb{P}^{3}$ be the blow-up of $\mathbb{P}^{3}$ with centre the transverse intersection $C=S_{1} \cap S_{2}$ of two smooth cubic surfaces $S_{1}$ and $S_{2}$. Then the strict transform $l$ of a normal twisted rational cubic curve meeting $C$ transversaly in exactly 8 points is a quasi-line of $X$, and a section of the projection $\varphi: X \rightarrow \mathbb{P}^{1}$ corresponding to the pencil defined by $S_{1}$ and $S_{2}$.

(2) Let $\psi: X \rightarrow \mathcal{Q}_{3} \subset \mathbb{P}^{4}$ be the blow-up of the 3-dimensional quadric $\mathcal{Q}_{3}$ with centre the transverse intersection $C=S_{1} \cap S_{2}$ of two smooth members $S_{1}$ and $S_{2}$ of $\left|\mathcal{O}_{\mathcal{Q}_{3}}(2)\right|$. Then the strict transform $l$ of a normal twisted rational cubic curve meeting $C$ transversally in exactly 5 points is a quasi-line of $X$, and a section of the projection $\varphi: X \rightarrow \mathbb{P}^{1}$ corresponding to the pencil defined by $S_{1}$ and $S_{2}$.

(3) Let $V_{3}$ be a smooth cubic hypersurface of $\mathbb{P}^{4}$, and let $\omega \subset V_{3}$ be a normal twisted rational cubic contained in $V_{3}$. Denote by $P_{3}$ the hyperplane in $\mathbb{P}^{4}$ generated by $\omega$. Let finally $l^{\prime}$ be a chord of $\omega$ (i.e. a line in $\mathbb{P}^{4}$ meeting $\omega$ in two points) and $P_{1}$ and $P_{2}$ be two general hyperplanes in $\mathbb{P}^{4}$ such that $l^{\prime}=P_{1} \cap P_{2} \cap P_{3}$. Let $\psi: X \rightarrow V_{3}$ be the blow-up of $V_{3}$ with centre $C=V_{3} \cap P_{1} \cap P_{2}\left(C\right.$ is therefore a plane cubic). Since $\omega \cap C \subset V_{3} \cap l^{\prime} \subset \omega \cap l^{\prime}$, the curve $\omega$ meets $C$ in exactly two points. The strict transform of $\omega$ is a quasiline of $X$, section of the projection $\varphi: X \rightarrow \mathbb{P}^{1}$ corresponding to the pencil defined by $V_{3} \cap P_{1}$ and $V_{3} \cap P_{2}$.

\subsection{Conic bundles}

The variety $\mathbb{P}\left(T_{\mathbb{P}^{2}}\right)$ is a Fano threefold that contains a quasi-line $l$ such that the natural projection to $\mathbb{P}^{2}$ induces a morphism of models $\left(\mathbb{P}\left(T_{\mathbb{P}^{2}}\right), l\right) \rightarrow\left(\mathbb{P}^{2}\right.$, line $)$. One can obtain conic bundles with the same property by blowing-up $\mathbb{P}\left(T_{\mathbb{P}^{2}}\right)$ along a smooth curve, but such a manifold would of course not be minimal. We will now show that this is what always happens.

Theorem 4.14. Let $\varphi:(X, l) \rightarrow(Y, \varphi(l))$ be a morphism of smooth models, and suppose that $\varphi$ is flat of relative dimension 1. Assume furthermore that:

(1) $X$ is minimal with respect to $l$ and $e(X, l)=1$,

(2) there exists a big and nef line bundle $H$ on $Y$ such that $\varphi^{*} H \cdot l=1$.

Then $Y \simeq \mathbb{P}^{n-1}$ and $X \simeq \mathbb{P}(E)$, where $E$ is a rank two vector bundle over $Y$. 
Proof. Since $X$ is minimal with respect to $l$, the manifold $Y$ is minimal with respect to $\varphi(l)$. By assumption (2) and Theorem 1.5, we get that $Y \simeq \mathbb{P}^{n-1}$ and $\varphi(l)$ is a line. Suppose first that $\varphi$ is a smooth morphism, so all the fibres are isomorphic to $\mathbb{P}^{1}$. Therefore $\varphi$ is locally analytically trivial and since the Brauer group of $\mathbb{P}^{n-1}$ vanishes, we see that $X$ is the projectivisation of a rank two bundle.

We will now argue by contradiction and suppose that $\varphi$ is not a smooth morphism. We claim that in this case there exists an effective divisor $\Delta$ in $Y$ such that the general $\varphi$-fibre over $\Delta$ is reducible. Since the general fibre is a rational curve [12, Theorem 1.12], this is clear if $\varphi$ is elementary (that is $\rho(X)-\rho(Y)=1$ ): $\varphi$ is a conic bundle and the discriminant locus is such a $\Delta$. If $\rho(X)-\rho(Y)>1$, just apply the relative contraction theorem [14, Theorem 4-1-1] to get an elementary contraction $\mu: X \rightarrow X^{\prime}$ that is a $Y$-morphism. It is not hard to see that $\mu$ is birational and by flatness of $\varphi$, all the fibres of $\mu$ have dimension at most 1 , so $X^{\prime}$ is smooth and, by Ando's theorem [1, Theorem 2.1], the morphism $\mu$ is the blow-up of a smooth codimension 2 subvariety $Z$ such that the image of $Z$ in $Y$ is a divisor. In particular there exists a divisor $\Delta \subset Y$ such that the general fibre over $\Delta$ is reducible.

Fix now an irreducible divisor $\Delta \subset Y$ such that the general $\varphi$-fibre over $\Delta$ is reducible, fix also a general point $x \in X$ such that $x \notin \varphi^{-1}(\Delta)$. We consider the deformations of $l$ with fixed point $x$. Recall the notation of the basic Diagram 2.1.

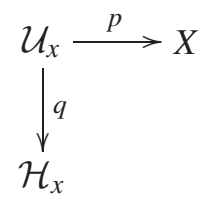

By Lemma 2.3, we have reached a contradiction if we show that an irreducible component $D \subset \varphi^{-1}(\Delta)$ is covered by irreducible components of degenerations with fixed point $x$.

Let $y$ be a general point of $\Delta$, then the fibre $\varphi^{-1}(y)$ has at least one singular point $z$ at the intersection of two components. Since $\varphi^{*} H \cdot l=1$, every quasiline $l$ parametrised by $\mathcal{H}_{x}$ is a subsection of $\varphi$, in particular it does not meet the singular points of fibres. Since the map $p$ is surjective, this shows that there exists a degeneration $l \sim \sum_{i=1}^{k} \alpha_{i} l_{i}$ that connects $x$ to $z$. Since $\varphi^{*} H \cdot \sum_{i=1}^{k} \alpha_{i} l_{i}=1$, this cycle can be written $l_{1}+\sum_{i \geq 2}^{k} \alpha_{i} l_{i}$ with $\varphi^{*} H \cdot l_{1}=1$ and $\varphi^{*} H \cdot l_{i}=0$ for $i \geq 2$.

Since $\varphi^{*} H \cdot l_{1}=1$, the curve $l_{1}$ is a subsection of a line, so it does not pass through $z$. Thus there exists a $j \geq 2$ such that $z \in l_{j}$. Since $\varphi^{*} H \cdot l_{j}=0$, this implies $l_{j} \subset \varphi^{-1}(\varphi(z))=\varphi^{-1}(y)$. We have shown that for a general point $y \in \Delta$, there exists a component of a degeneration of $l$ contained in $\varphi^{-1}(y)$. Therefore there exists at least one irreducible component of $\varphi^{-1}(\Delta)$ that is dominated by irreducible components of degenerations of $l$. Lemma 2.3 shows that this is a contradiction. 
The following examples show the importance of the assumption that $e(X, l)=1$ for the theorem.

\section{Examples 4.15.}

(1) Let $X$ be a double cover of $\mathbb{P}^{1} \times \mathbb{P}^{2}$ whose branch locus is a general divisor of bidegree $(2,2)$. The threefold $X$ is Fano with Picard number 2 , denote by $\psi: X \rightarrow \mathbb{P}^{1}$ and $\varphi: X \rightarrow \mathbb{P}^{2}$ the two natural projections. The map $\varphi$ is a conic bundle, whose discriminant locus is a quartic curve in $\mathbb{P}^{2}$ and the map $\psi$ is a quadric bundle. Let $l$ be a general line in $\mathbb{P}^{2}$ and set $S_{l}:=\varphi^{-1}(l)$. The surface $S_{l}$ is a del Pezzo surface and the induced map $\varphi: S_{l} \rightarrow l \simeq \mathbb{P}^{1}$ has exactly 4 singular fibres. More precisely, we have a diagram

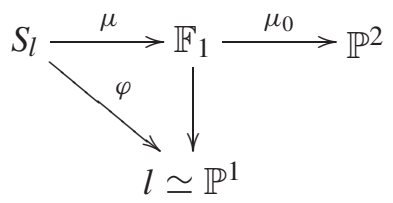

where $\mu_{0}$ is the blow-up of a point $x_{0} \in \mathbb{P}^{2}$ and $\mu$ is the blow-up of 4 points $p_{1}, \ldots, p_{4}$ in different fibres. Set $\pi:=\mu_{0} \circ \mu: S_{l} \rightarrow \mathbb{P}^{2}$, and let $d$ be a general line in $\mathbb{P}^{2}$ and $\omega=\pi^{-1}(d)$. Clearly $\omega$ is a quasi-line of $S_{l}$ and a section of $\varphi: S_{l} \rightarrow l$, therefore $\omega$ is a quasi-line of $X$ by Lemma 4.1. One sees that $(X, \omega)$ satisfies all hypothesis of Theorem 4.14 except $e(X, \omega)=1$ : if $x$ and $x^{\prime}$ are two general points of $\omega$, take a cubic curve in $P$, passing through $x, x^{\prime}$, $\mu_{0}\left(p_{i}\right)$ for $i=1, \ldots, 4$ with multiplicity 1 , and through $x_{0}$ with multiplicity 2 . Then the strict transform of this cubic is also a quasi-line of $S_{l}$ and a section of $\varphi: S_{l} \rightarrow l$, hence is also a quasi-line of $X$ by Lemma 4.1 .

Note that these two quasi-lines do not belong to the same family as quasi-lines of $S_{l}$ but belong to the same family as quasi-lines of $X$ !

(2) Exactly in the same manner, let $X$ be a double cover of $\mathbb{P}^{1} \times \mathbb{P}^{2}$ whose branch locus is a divisor of bidegree $(2,4)$. The threefold $X$ is Fano with Picard number 2 , denote by $\psi: X \rightarrow \mathbb{P}^{1}$ and $\varphi: X \rightarrow \mathbb{P}^{2}$ the two natural projections. The map $\varphi$ is a conic bundle, whose discriminant is a curve of degree 8 in $\mathbb{P}^{2}$ and the map $\psi$ is a del Pezzo fibration. Let $l$ be a general line in $\mathbb{P}^{2}$ and $S_{l}:=\varphi^{-1}(l)$. The surface $S_{l}$ is a surface with nef anti-canonical bundle (isomorphic to a $\mathbb{P}^{2}$ blown-up at 9 general points) and the induced map $\varphi: S_{l} \rightarrow l \simeq \mathbb{P}^{1}$ has exactly 8 singular fibres. One can show that $S_{l}$ contains a quasi-line, section of $\varphi: S_{l} \rightarrow l$, hence a quasi-line of $X$.

\section{Fano bundles}

Theorem 4.14 shows that projective bundles over $\mathbb{P}^{m}$ play a special role in the classification of models $(X, l)$ with $e(X, l)=1$, in particular if $X$ is minimal with 
respect to $l$. More generally we might ask when a Fano manifold that is a projectivised bundle $\mathbb{P}(E)$ (a so-called Fano bundle) over $\mathbb{P}^{m}$ contains a quasi-line. Classification results and vector bundle techniques will allow us to give a complete answer if $\mathrm{rk} E=2$. Together with Theorem 4.14, this also ends the proof of Theorem C.

Notation. Let $\varphi: \mathbb{P}(E) \rightarrow \mathbb{P}^{m}$ be a projectivised bundle over $\mathbb{P}^{m}$ (we use here Grothendieck's definition: $\mathbb{P}(E)$ is the variety of hyperplanes of $E$ ). Throughout the whole section, we will denote by $H$ a hyperplane divisor in $\mathbb{P}^{m}$, and by $\xi_{E}$ the tautological divisor on $\mathbb{P}(E)$. If $l \subset \mathbb{P}(E)$ is a quasi-line, we will use frequently $\varphi^{*} H \cdot l \geq 1$.

\section{Proposition 5.1.}

1. Let $(X, l)$ be a smooth model, and suppose that $X$ is minimal with respect to $l$. Suppose furthermore that $X \simeq \mathbb{P}(E)$ for some rank 2 vector bundle $E$ over the projective space $\mathbb{P}^{m}$. Then the vector bundle $E$ is stable.

2. Let $E$ be a semistable vector bundle of rank 2 with odd first Chern class over the projective space $\mathbb{P}^{m}$. Then $X \simeq \mathbb{P}(E)$ contains a quasi-line $l$ and the natural projection $\varphi: X \rightarrow \mathbb{P}^{m}$ is a morphism of models $(X, l) \rightarrow\left(\mathbb{P}^{m}\right.$, line $)$.

Proof. For the first statement, we argue by contradiction and suppose that $E$ is not stable. Then, up to twisting $E$ with a line bundle, we can suppose without loss of generality that $-1 \leq c_{1}(E) \leq 0$ and $h^{0}(X, E)>0$ (cf. [9, Remark 3.0.1]). Let $\varphi: X \rightarrow \mathbb{P}^{m}$ be the projection map, then

$$
-K_{X}=\left(m+1-c_{1}(E)\right) \varphi^{*} H+2 \xi_{E} .
$$

It follows that

$$
m+2=-K_{X} \cdot l=\left(m+1-c_{1}(E)\right) \varphi^{*} H \cdot l+2 \xi_{E} \cdot l \geq m+1+2 \xi_{E} \cdot l .
$$

Since $h^{0}\left(X, \xi_{E}\right)=h^{0}\left(\mathbb{P}^{m}, E\right)>0$ and $X$ is minimal, we have $\xi_{E} \cdot l \geq 1$. This implies

$$
m+2 \geq m+1+2 \xi_{E} \cdot l \geq m+1+2
$$

a contradiction.

For the second statement, let $l^{\prime} \subset \mathbb{P}^{m}$ be a general line. By the Grauert-Mülich theorem [19, page 206], we have $\left.E\right|_{l^{\prime}} \simeq \mathcal{O}_{\mathbb{P}^{1}}\left(a_{1}\right) \oplus \mathcal{O}_{\mathbb{P}^{1}}\left(a_{2}\right)$ with $\left|a_{2}-a_{1}\right| \leq 1$. Since $E$ has an odd first Chern class, we can suppose up to renumbering that $a_{2}=a_{1}+1$. By [12, Proposition 4.2] there exists a quasi-line $l$ on $X$ such that $\left.\varphi\right|_{l}: l \rightarrow l^{\prime}$ is an isomorphism.

Remark 5.2. By a well-known conjecture of Hartshorne [7], there are no stable rank 2 vector bundles over $\mathbb{P}^{m}$ if $m \geq 7$. This shows (at least conjecturally) that the minimality with respect to a quasi-line is rather restrictive. Note however that the fourfold $\mathbb{P}\left(T_{\mathbb{P}^{2}}(-1) \oplus \mathcal{O}_{\mathbb{P}^{2}}\right)$ contains a quasi-line and is minimal, but the vector bundle $T_{\mathbb{P}^{2}}(-1) \oplus \mathcal{O}_{\mathbb{P}^{2}}$ isn't even semistable. This shows that for a generalisation of Proposition 5.1 to bundles of higher rank new ideas are necesssary. 
Proposition 5.3. Let $E \simeq \oplus_{i=1}^{r+1} \mathcal{O}_{\mathbb{P}^{m}}\left(a_{i}\right)$ be a sum of line bundles on $\mathbb{P}^{m}$ such that $a_{1} \leq a_{2} \leq \ldots \leq a_{r+1}$. Set $X:=\mathbb{P}(E)$, then $X$ contains a quasi-line $l$ if and only if up to twisting

$$
E \simeq \mathcal{O}_{\mathbb{P}^{m}}(-1)^{\oplus r} \oplus \mathcal{O}_{\mathbb{P}^{m}}
$$

In this case the natural map $\varphi: X \rightarrow \mathbb{P}^{m}$ is a morphism of models $(X, l) \rightarrow$ $\left(\mathbb{P}^{m}\right.$, line $)$.

Proof. The if part is immediate from [12, Proposition 4.2], for the only if part we follow closely the proof of [12, Proposition 4.1]. We have

$$
-K_{X}=\left(m+1-\sum_{i=1}^{r+1} a_{i}\right) \varphi^{*} H+(r+1) \xi_{E} .
$$

and

$$
h^{0}\left(X, \mathcal{O}_{X}\left(\xi_{E}-a_{r+1} \varphi^{*} H\right)\right)=h^{0}\left(\mathbb{P}^{m}, E \otimes \mathcal{O}_{\mathbb{P}^{m}}\left(-a_{r+1}\right)\right)>0,
$$

so the divisor $\xi_{E}-a_{r+1} \varphi^{*} H$ is linearly equivalent to an effective divisor. In particular

$$
\left(\xi_{E}-a_{r+1} \varphi^{*} H\right) \cdot l \geq 0 .
$$

We want to show that even $\left(\xi_{E}-a_{r+1} \varphi^{*} H\right) \cdot l=0$ and argue by contradiction. Then

$$
\left(\xi_{E}-a_{i} \varphi^{*} H\right) \cdot l \geq\left(\xi_{E}-a_{r+1} \varphi^{*} H\right) \cdot l \geq 1
$$

for all $i=1, \ldots r+1$, so

$$
\begin{aligned}
m+r+1=-K_{X} \cdot l & =\left(m+1-\sum_{i=1}^{r+1} a_{i}\right) \varphi^{*} H \cdot l+(r+1) \xi_{E} \cdot l \\
& =(m+1) \varphi^{*} H \cdot l+\sum_{i=1}^{r+1}\left(\xi_{E}-a_{i} \varphi^{*} H\right) \cdot l \\
& \geq(m+1)+(r+1)
\end{aligned}
$$

yields a contradiction. So $\left(\xi_{E}-a_{r+1} \varphi^{*} H\right) \cdot l=0$ and

$$
h^{0}\left(X, \mathcal{O}\left(\xi_{E}-a_{r+1} \varphi^{*} H\right)\right)=h^{0}\left(\mathbb{P}^{m}, E \otimes \mathcal{O}\left(-a_{r+1}\right)\right)=1,
$$

since a quasi-line has strictly positive intersection number with every divisor that moves. This implies that $a_{i}<a_{r+1}$ for $i=1, \ldots, r$, so

$$
\left(\xi_{E}-a_{i} \varphi^{*} H\right) \cdot l>\left(\xi_{E}-a_{r+1} \varphi^{*} H\right) \cdot l=0
$$

for all $i=1, \ldots r$. We repeat the preceeding computation

$$
\begin{aligned}
m+r+1=-K_{X} \cdot l & =(m+1) \varphi^{*} H \cdot l+\sum_{i=1}^{r+1}\left(\xi_{E}-a_{i} \varphi^{*} H\right) \cdot l \\
& \geq(m+1) \varphi^{*} H \cdot l+r,
\end{aligned}
$$


so $\varphi^{*} H \cdot l=1$. This implies that $\varphi(l)$ is a line in $\mathbb{P}^{m}$ and $\left.\varphi\right|_{l}: l \rightarrow \varphi(l)$ is an isomorphism. We conclude with a second application of [12, Proposition 4.2].

Corollary 5.4. Let $(X, l)$ be a smooth Fano model. Suppose that $X \simeq \mathbb{P}(E)$ for some rank 2 vector bundle $E$ over the projective space $\mathbb{P}^{m}$ with $m \geq 3$. Then up to twisting with a line bundle we have

$$
E \simeq \mathcal{O}_{\mathbb{P}}(-1) \oplus \mathcal{O}_{\mathbb{P}^{m}}
$$

so $X$ is the blow-up of $\mathbb{P}^{m+1}$ in a point and $l$ is the preimage of a line in $\mathbb{P}^{m+1}$. In particular $X$ is not minimal.

\section{Proof.}

1st case: $m \geq 4$. Since $E$ is a rank 2 Fano bundle, it splits by [2, Main theorem] in a direct sum of line bundles. Conclude with Proposition 5.3.

2nd case: $m=3$. We normalise $E$ such that $c_{1}(E)=0$ or $c_{1}(E)=-1$. If the first Chern class is zero, we have

$$
-K_{X}=4 \varphi^{*} H+2 \xi_{E}
$$

In particular $X$ has index 2, so it is clear that $X$ does not contain a quasi-line $\left(-K_{X} \cdot l=5\right)$. If $c_{1}(E)=-1$, the classification of Fano bundles of rank 2 on $\mathbb{P}^{3}\left[21\right.$, Theorem 2.1], implies that $E \simeq \mathcal{O}_{\mathbb{P}^{3}} \oplus \mathcal{O}_{\mathbb{P}^{3}}(-1)$ or $E \simeq \mathcal{O}_{\mathbb{P}^{3}}(-2) \oplus \mathcal{O}_{\mathbb{P}^{3}}(1)$. The second case is excluded by Proposition 5.3.

Proposition 5.5. Let $(X, l)$ be a smooth Fano model. Suppose that $X \simeq \mathbb{P}(E)$ for some vector bundle $E$ of rank $r$ over the projective space $\mathbb{P}^{2}$. Then up to twisting with a line bundle we have either

$$
E \simeq \mathcal{O}_{\mathbb{P}^{2}}^{\oplus r-1} \oplus \mathcal{O}_{\mathbb{P}^{2}}(1)
$$

or $E$ is defined by an exact sequence

$$
0 \rightarrow \mathcal{O}_{\mathbb{P}^{2}}(-1)^{\oplus 2} \rightarrow \mathcal{O}_{\mathbb{P}^{2}}^{\oplus r+2} \rightarrow E \rightarrow 0
$$

or

$$
E \simeq T_{\mathbb{P}^{2}}(-1) \oplus \mathcal{O}_{\mathbb{P}^{2}}^{\oplus r-2}
$$

or $E$ is defined by an exact sequence

$$
0 \rightarrow \mathcal{O}_{\mathbb{P}^{2}}(-2) \rightarrow \mathcal{O}_{\mathbb{P}^{2}}^{r+1} \rightarrow E \rightarrow 0
$$

Vice versa, if $E$ is a vector bundle over $\mathbb{P}^{2}$ as above, then $\mathbb{P}(E)$ is Fano and contains a quasi-line.

In the first two cases there exists a birational morphism $X \rightarrow \mathbb{P}^{r+1}$ that induces an equivalence of models $(X, l) \simeq\left(\mathbb{P}^{r+1}\right.$, line $)$; in the last two cases $X$ is minimal with respect to $l$. In the third case $e(X, l)=1$ and in the fourth case $e(X, l)>1$. 
Proof. Denote by $\varphi: X \rightarrow \mathbb{P}^{2}$ the projection map. We normalise the vector bundle $E$ such that $0 \leq c_{1}(E) \leq r-1$, then by $[22,1.6$.] the Fano condition implies $0 \leq c_{1}(E) \leq 2$. Furthermore the vector bundle $E$ is globally generated [22, Proposition 2.1]. Since $E$ can't be trivial (the product $\mathbb{P}^{r-1} \times \mathbb{P}^{2} \simeq \mathbb{P}(E)$ does not contain a quasi-line), this implies

$$
\text { (*) } \quad h^{0}\left(X, \mathcal{O}_{X}\left(\xi_{E}\right)\right)=h^{0}\left(\mathbb{P}^{2}, E\right) \geq r+1,
$$

in particular

$$
\xi_{E} \cdot l \geq 1
$$

Therefore $\varphi^{*} \mathcal{O}_{\mathbb{P}^{2}}\left(3-c_{1}(E)\right) \cdot l \geq 1$ implies

$$
\begin{aligned}
r+2 & =-K_{X} \cdot l \\
& =\left(3-c_{1}(E)\right) \varphi^{*} H \cdot l+r \xi_{E} \cdot l \\
& \geq 1+r \xi_{E} \cdot l .
\end{aligned}
$$

Hence $\xi_{E} \cdot l=1$, since $\mathcal{O}_{X}\left(\xi_{E}\right)$ is globally generated it induces by [12, Theorem 1.12] a surjective morphism with connected fibres $\psi: X \rightarrow \mathbb{P}^{N}:=\mathbb{P}\left(H^{0}\left(X, \mathcal{O}_{X}\left(\xi_{E}\right)\right)\right)$ that induces a morphism of models $(X, l) \rightarrow\left(\mathbb{P}^{N}\right.$, line $)$. In particular

$$
h^{0}\left(X, \mathcal{O}_{\mathbb{P}(E)}(1)\right)=h^{0}\left(\mathbb{P}^{2}, E\right) \leq \operatorname{dim} X+1=r+2
$$

and equality holds if and only if $\psi$ induces an equivalence of models $(X, l) \simeq\left(\mathbb{P}^{r+1}\right.$, line $)$. In view of the inequalities $(*)$ and $(* *)$ we have to treat two cases.

The case $h^{0}\left(\mathbb{P}^{2}, E\right)=r+2$. Going through the list in [22, Theorem ] yields that in this case, we have $E \simeq \mathcal{O}_{\mathbb{P}^{2}}^{\oplus r-1} \oplus \mathcal{O}_{\mathbb{P}^{2}}(1)$ or $E$ is defined by an exact sequence

$$
0 \rightarrow \mathcal{O}_{\mathbb{P}^{2}}(-1)^{\oplus 2} \rightarrow \mathcal{O}_{\mathbb{P}^{2}}^{\oplus r+2} \rightarrow E \rightarrow 0
$$

In both cases, the manifold $X$ is a blow-up of $\mathbb{P}^{r+1}$, so it is clear that $X$ contains a quasi-line and is not minimal with respect to it.

The case $h^{0}\left(\mathbb{P}^{2}, E\right)=r+1$. In this case $\psi$ gives a fibration of relative dimension one onto $\mathbb{P}^{r}$ that induces a morphism of models $(X, l) \rightarrow\left(\mathbb{P}^{r}\right.$, line $)$. Using the list in $\left[22\right.$, Theorem ], we then see that $E \simeq T_{\mathbb{P}^{2}}(-1) \oplus \mathcal{O}_{\mathbb{P}^{2}}^{\oplus r-2}$ or $E$ is defined by an exact sequence

$$
0 \rightarrow \mathcal{O}_{\mathbb{P}^{2}}(-2) \rightarrow \mathcal{O}_{\mathbb{P}^{2}}^{\oplus r+1} \rightarrow E \rightarrow 0 .
$$

In the first case $c_{1}(E)=1$, so

$$
r+2=-K_{X} \cdot l=2 \varphi^{*} H \cdot l+r .
$$


implies that $\varphi^{*} H \cdot l=1$. Hence $\varphi(l)$ is a line in $\mathbb{P}^{2}$ and $l$ is a section. Vice versa [12, Proposition 4.2] shows that $\mathbb{P}\left(T_{\mathbb{P}^{2}}(-1) \oplus \mathcal{O}_{\mathbb{P}^{2}}^{\oplus r-2}\right)$ contains a quasi-line $l$ that is a subsection of a line in $\mathbb{P}^{2}$. Since the two elementary contractions are of fibre type, the manifold $X$ is minimal with respect to $l$ by Lemma 3.1. The equality $e(X, l)=1$ follows from the remark below.

In the second case $X$ can be realised as a smooth divisor of degree $(2,1)$ in $\mathbb{P}^{2} \times \mathbb{P}^{r}$ [22, Theorem], and $\varphi$ (respectively $\psi$ ) identifies to the projection on the first (respectively second) factor. Since the property of containing a quasi-line is stable under small deformations [3, Proposition 3.10], we can choose $X$ sufficiently general such that $\psi$ has no higher-dimensional fibres, i.e. yields a conic bundle structure on $X$ and a straightforward computation shows that the discriminant locus has degree 3. By adjunction $\omega_{X}^{*} \simeq \mathcal{O}_{X}(1, r)$, so if $l^{\prime} \subset \mathbb{P}^{r}$ is a general line and $S:=\psi^{-1}\left(l^{\prime}\right)$ is its preimage, repeated adjunction yields $\omega_{S}^{*} \simeq \mathcal{O}_{S}(1,1)$. Hence $S$ is a del Pezzo surface and $\left.\psi\right|_{S}: S \rightarrow l^{\prime}$ has three singular fibres. The surface $S$ is a blow-up of $\mathbb{P}^{2}$ in four points, so a general line in $\mathbb{P}^{2}$ yields a quasi-line $l$ in $S$ that is a section of $\left.\psi\right|_{S}$. By Lemma 4.1 this shows that $l$ is a quasi-line in $X$. Since the two elementary contractions are of fibre type, the manifold $X$ is minimal with respect to $l$ by Lemma 3.1. Since $\psi$ is not a smooth morphism, Theorem 4.14 shows that $e(X, l)>1$.

Remark 5.6. Let $\varphi:(X, l) \rightarrow(Y, \varphi(l))$ be a morphism of smooth models that is a fibration. Choose a sufficiently general deformation of $l$ such that $Z:=\varphi^{-1}(\varphi(l))$ is smooth. We define

$$
e(Z):=\sum_{l \in \mathcal{H}} e(X, l)
$$

where the sum goes over all the irreducible components of the Chow scheme such that the general point parametrises a quasi-line. Then it is not hard to check (see [11, Lemma 5.9] for the special case of a projective bundle) that

$$
e(X, l) \leq e(Z) \cdot e(Y, \varphi(l)) .
$$

In particular if $X \simeq \mathbb{P}(E)$ for a vector bundle $E$ of rank $r$ over $Y$, then $Z \simeq \mathbb{P}\left(\mathcal{O}_{\mathbb{P}_{1}}^{\oplus r-1} \oplus \mathcal{O}_{\mathbb{P}_{1}}(1)\right)[12$, Proposition 4.1, Proposition 4.2], so $e(Z)=1$ and $e(X, l) \leq e(Y, \varphi(l))$.

The fourth case of the proposition shows that not all the Fano bundles over $\mathbb{P}^{2}$ satisfy $e(X, l)=1$. In particular $X \rightarrow \mathbb{P}^{2}$ is not a morphism of models!

Towards a classification of Fano models? It is an obvious question to ask for a complete list of Fano models $(X, l)$ such that $X$ is minimal with respect to $l$. This turns out to be a rather lengthy exercice, in fact using the techniques in this paper (in particular the Examples 4.15) one can show that out of the nine primitive Fano threefolds with Picard number two, all but $\mathbb{P}^{2} \times \mathbb{P}^{1}$ and $\mathbb{P}\left(\mathcal{O}_{\mathbb{P}^{2}} \oplus \mathcal{O}_{\mathbb{P}^{2}}(2)\right)$ contain a quasi-line. Among these models, five have only fibre type contractions, so they are minimal with respect to the quasi-line (Lemma 3.1). Subsection 4.2 
shows how to obtain more examples in the non-primitive case. This convinces us that classification efforts should concentrate on the case $e(X, l)=1$. In this case we only know one example with Picard number at least two: the flag manifold $\mathbb{P}\left(T_{\mathbb{P}^{2}}\right)$.

\section{References}

[1] T. ANDo, On extremal rays of the higher-dimensional varieties, Invent. Math. 81 (1985), 347-357.

[2] V. Ancona, T. Peternell and J. A. Wiśniews Ki, Fano bundles and splitting theorems on projective spaces and quadrics, Pacific J. Math. 163 (1994), 17-42.

[3] L. BĂdescu, M. C. Beltrametti and P. Ionescu, Almost-lines and quasi-lines on projective manifolds, In: "Complex analysis and algebraic geometry", de Gruyter, Berlin, 2000, 1-27.

[4] K. Cho, Y. MiYaoka and N. I. ShePherd-BARron, Characterizations of projective space and applications to complex symplectic manifolds, In: "Higher dimensional birational geometry" (Kyoto, 1997), Adv. Stud. Pure Math., Vol. 35, Math. Soc. Japan, Tokyo, 2002, $1-88$.

[5] O. DebarRe, "Higher-Dimensional Algebraic Geometry", Universitext, Springer-Verlag, New York, 2001.

[6] T. FuJITA, On polarized manifolds whose adjoint bundles are not semipositive, In: "Algebraic geometry" (Sendai, 1985), Adv. Stud. Pure Math., Vol. 10, North-Holland, Amsterdam, 1987, 167-178.

[7] R. HARTSHORne, Varieties of small codimension in projective space, Bull. Amer. Math. Soc. 80 (1974), 1017-1032.

[8] R. Hartshorne, "Algebraic Geometry", Graduate Texts in Mathematics, Vol. 52. Springer-Verlag, New York, 1977.

[9] R. Hartshorne, Stable vector bundles of rank 2 on $\mathbf{P}^{3}$, Math. Ann. 238 (1978), 229-280.

[10] N. J. Hitchin, Kählerian twistor spaces, Proc. London Math. Soc. 43 (1981), 133-150.

[11] P. IONESCU and D. NAIE, Rationality properties of manifolds containing quasi-lines, Internat. J. Math. 14 (2003), 1053-1080.

[12] P. IONESCU and C. VoICA, Models of rationally connected manifolds, J. Math. Soc. Japan 55 (2003), 143-164.

[13] S. KebeKus, Characterizing the projective space after Cho, Miyaoka and ShepherdBarron, In: "Complex geometry" (Göttingen, 2000), Springer, Berlin, 2002, 147-155.

[14] Kawamata, Yujiro, Matsuda, Katsumi and Matsuki, Kenji, Introduction to the minimal model problem, In: "Algebraic Geometry" (Sendai, 1985), Adv. Stud. Pure Math., Vol. 10, North-Holland, Amsterdam, 1987, 283-360.

[15] J. KolláR, "Rational Curves on Algebraic Varieties", Ergebnisse der Mathematik und ihrer Grenzgebiete, 3. Folge. A Series of Modern Surveys in Mathematics, Vol. 32, SpringerVerlag, Berlin, 1996.

[16] S. Mori and S. MukaI, Classification of Fano 3-folds with $B_{2} \geq 2$, Manuscripta Math. 36 (1981/82), 147-162.

[17] S. MORI and S. MUKAI, On Fano 3-folds with $B_{2} \geq 2$, In: "Algebraic varieties and analytic varieties" (Tokyo, 1981), Adv. Stud. Pure Math. Vol. 1, North-Holland, Amsterdam, 1983, 101-129.

[18] S. MORI, Threefolds whose canonical bundles are not numerically effective, Ann. of Math. 116 (1982), 133-176.

[19] C. OkoneK, M. Schneider and H. Spindler, "Vector Bundles on Complex Projective Spaces", Progress in Mathematics, Vol. 3, Birkhäuser Boston, Mass., 1980.

[20] W. M. OXBURY, Twistor spaces and Fano threefolds, Quart. J. Math. Oxford Ser. 45 (1994), 343-366. 
[21] M. SzUreK and J. A. Wiśniewski, Fano bundles over $\mathbf{P}^{3}$ and $Q_{3}$, Pacific J. Math. 141 (1990), 197-208.

[22] M. SZUREK and J. A. WiŚNIEWski, On Fano manifolds, which are $\mathbf{P}^{k}$-bundles over $\mathbf{P}^{2}$, Nagoya Math. J. 120 (1990), 89-101.

[23] J. A. WIŚNIEWSKI, On contractions of extremal rays of Fano manifolds, J. Reine Angew. Math. 417 (1991), 141-157.

Institut Fourier, UMR 5582

Université de Grenoble 1, BP 74

38402 Saint Martin d'Hères, France

laurent.bonavero@ujf-grenoble.fr

Université Pierre et Marie Curie

Institut de Mathématiques de Jussieu

Equipe de Topologie et Géométrie Algébrique 175, rue du Chevaleret

75013 Paris, France

hoering@math.jussieu.fr 